\title{
Colocutanous fistula after percutanous drainage in a patient with diverticular disease
}

\author{
Mehmet Yildirim* and Savas Yakan \\ Bozyaka Education and Research Hospital, Depertmant of General Surgery, University of Health Sciences, Izmir, Turkey
}

We would like to draw readers attention to colocutanous fistulas,notable rare in this letter. In this report, we present a patient with colonic diverticular disease Type III according to Hinchey classification mimicking subcutaneous abscess and despite effective antiinflammatory therapy and drainage resulting colocutanous fistula. However, internal fistulas of diverticular diseases are well-defined in literature, colocutaneous fistulas well not documanted after percutane drainage of a cutanous abscess [1-3].

A 63-year-old man with a faecal fistula in the left lower anterior abdominal region was admitted to our Department of Surgery. The patient's chief complaint is abdominal pain and low-output fecal purulent discharge from a fistula tract (Figure 1). Three days before presentation, the patient was felt colicky pain in the lower left abdominal quadrant. After the pain persisted and, on the following day, it intensified and was accompanied by a recorded fever of $38^{\circ} \mathrm{C}$. He has not noted any changes in his bowel movements. The patient has not taken any medication for the abdominal pain.
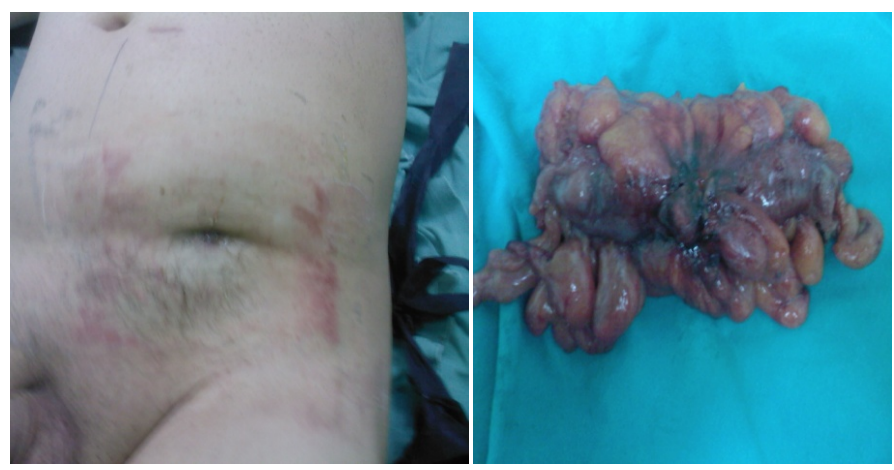

Figure 1.The appearance of fistula orifice at left and at right macroscopic appearance of the resected inflamed colon segment with perforated diverticulitis.
His past medical history included diabetes mellitus, and no significant any disease. He had percutaneous drainage with the diagnosis of subcutanous abscess 28 days ago in an Invaziv Radiology Section of a different hospital. CT showed peridiverticular abscess formation sprawling into the rectus abdominis muscle and subcutanous planes.

With these imaging findings in hand, we considered a colocutaneous fistula triggered by percutaneous drainage of an abscess originated perforated diverticular disease. As a result,we performed laparotomy which revealed a mass that sigmoid colon herniation originating from a inflamed diverticulitis through the posterior rectus sheath in the left lower abdominal wall and cutaneous fistula formation. We reduced the mass containing inflamed colon segment, and performed segmenter colon resection with end-to-end colocolic anastomosis. We curetted fistula tract, and sutured the posterior fascia of the rectus muscle. The patient was discharged on the 7 th postoperative day of the surgery. At followup examination after three months the patient was in good condition. Histologic examination of the speciman showed no malignancy.

In our view,differential diagnosis is an important entity for abdominal skin abscess. We think that, surgical resection of fistulised segment is reserved for those Grade III diverticulitis. Drainage with imaging studies must be applied to prevent unwanted local and systemic complications.

\section{References}

1. Woods RJ, Laveny IC, Fazio VW, Jagelman DG, Weakley FL (1988) Internal fistulas in diverticular disease. Dis Colon Rectum 31: 591-596. [Crossref]

2. Singh B, May K, Coltart I, Moore NR, Cunningham C (2008) The long-term results of percutaneous drainage of diverticlar abscess. Ann R Coll Surg Engl 90: 297-301. [Crossref]

3. Ambrosetti P, Robert J, Witzig JA, Mirescu D, De Gautard R, et al. (1992) Incidence, outcome, and proposed management of isolated abscesses complicating acute left-sided colonic diverticulitis. A prospective study of 140 patients. Dis Colon Rectum 35: 10721076. [Crossref]
Copyright: (C2017 Yildirim M. This is an open-access article distributed under the terms of the Creative Commons Attribution License, which permits unrestricted use, distribution, and reproduction in any medium, provided the original author and source are credited.
Correspondence to: Mehmet Yildirim, Bozyaka Education and Research Hospital, Depertmant of General Surgery, University of Health Sciences, Izmir, Turkey, Tel. 090-532-2855557; E-mail: mehmetyildi@gmail.com

Received: August 21, 2017; Accepted: September 18, 2017; Published: September 21, 2017 